JIOM Nepal, Volume 41, Number 2, August 2019, page 21-25

\title{
Nutritional Assessment of Patients with Liver Cirrhosis by Nutrition Screening Tool and Anthropometry at a Tertiary Care Center
}

\author{
Tshering W Sherpa, Rahul Pathak, Prem K Khadga, Sashi Sharma, Rabin Hamal, Anurag Jha \\ Department of Gastroenterology, Maharajgunj Medical Campus, Institute of Medicine, Tribhuvan University Teaching \\ Hospital

\section{Corresponding author:} \\ Tshering W Sherpa, MBBS, MD \\ Department of Gastroenterology, Maharajgunj Medical Campus, Institute of Medicine, Tribhuvan University Teaching \\ Hospital \\ Email: tshering.ongdi@gmail.com
}

Submitted: May 23, 2019

Accepted : July 25, 2019

\begin{abstract}
Introduction

Malnutrition is one of the most common complications of liver cirrhosis. Yet, little attention is paid in evaluating nutrition in this group of patients. This study aims to assess malnutrition among cirrhotic patients using a nutrition screening tool and anthropometry.

\section{Methods}

This was a prospective, observational study of admitted patients with liver cirrhosis. In the study duration of 3 months, 50 patients met the inclusion criteria and were included. Nutritional assessment was performed using the Royal Free Hospital Nutritional Prioritizing Tool (RFH-NPT), BMI and standard anthropometry including TSF, MUAC and MAMC.

\section{Results}

The mean age was $51.56 \pm 11.50$ with a Male to Female ratio of 3:2. Chronic alcohol consumption (72\%) was the most common etiology while management of tense ascites (40\%) was the most common reason for hospital admission. $58 \%$ had Child Pugh Class C cirrhosis while the remaining $42 \%$ were Class $B$. The average MELD Na score was $19.64 \pm 6$. Significant differences in anthropometric measurements including BMI, MUAC, TSF and MAMC were found between Child B and C cirrhosis. Similarly, those patients who had low, moderate and high risk of malnutrition by the RFH-NPT had significant differences in anthropometric measurements between them.

\section{Conclusion}

A significant number of patients had moderate to severe risk of malnutrition that correlated well with anthropometric measurements. The degree of malnutrition is parallel with the severity of liver disease among these patients. Both the RFH-NPT and anthropometry are relatively easy to perform and effective. Hence, they can be used as a practical means for identifying malnutrition among cirrhotic patients in routine clinical practice.
\end{abstract}

Keywords: Anthropometry, liver cirrhosis, malnutrition

\section{INTRODUCTION}

$$
\square
$$

iver cirrhosis, characterized by fibrosis and architectural distortion of the hepatic parenchyma that leads to portal hypertension and its related complications is a common cause of morbidity and mortality worldwide. Malnutrition is one of the most common complications of liver cirrhosis but is often overlooked. The worldwide prevalence of malnutrition among cirrhotic patients range from 50 to $99 \%$ depending upon the different assessment methods used.1,2 Excess alcohol consumption, the most common cause of liver cirrhosis in our context also contributes to malnutrition. A study including 536 male veterans with a clinical diagnosis of alcoholic hepatitis, showed that almost all patients with alcohol related liver disease had some level of protein calorie malnutrition and the degree of malnutrition was associated with survival at six months. ${ }^{3}$ Malnutrition is also associated with the severity of the disease among hospitalized patients with cirrhosis $^{4,5}$ and other complications such as ascites ${ }^{5}$ and bacterial infections. ${ }^{6}$ Similarly, the loss of skeletal muscle mass hampers the removal of ammonia from the circulation which further contributes to hepatic encephalopathy in these patients. ${ }^{1}$

Nutritional assessment in patients with liver cirrhosis 
is difficult due to presence of fluid overload, sex differences in body composition and lack of validated tools. As of now, there are no specific guidelines for nutritional assessment in these patients. However, it is recommended that all patients with liver cirrhosis undergo a rapid nutritional screening test to determine the risk of malnutrition. Those who are at risk should then be subject to a more detailed nutritional assessment to confirm the presence and severity of malnutrition. ${ }^{7-9}$ A nutritional screening tool should be simple, quick and reproducible that can be performed by nonexperts as well with a relatively good sensitivity and specificity. ${ }^{10}$ The Royal Free Hospital Nutrition Prioritizing tool (RFHNPT) is one such tool that is simple to perform with excellent intra-and inter-observer reproducibility and has been validated in a UK multicenter trial. ${ }^{11}$ It has also been shown that the RFH-NPT correlates well with severity of the disease and portal hypertension related complications such as ascites, hepatorenal syndrome and hepatic encephalopathy. ${ }^{1,12}$ Though not very sensitive, Body Mass Index (BMI) can be used to assess malnutrition after correcting the weight for the severity of ascites and peripheral edema. ${ }^{7}$.13 Anthropometric measurements including Mid Upper Arm Circumference (MUAC), TST (Triceps Skin Fold Thickness) and Mid Arm Muscle Circumference (MAMC) are simple and quick to perform with good inter-observer agreement and are probably the most practically applicable methods. In addition, TST and MAMC have demonstrated a linear relationship with mortality among cirrhotic patients. ${ }^{14}$

The primary aim of this study is to determine the nutritional status of Nepalese patients admitted to the hospital using the RFH-NPT and standard anthropometry.

\section{METHODS}

This was a prospective, observational study conducted among patients with liver cirrhosis and portal hypertension related complications admitted to the gastroenterology department of TUTH. Diagnosis was based on clinical, biochemical, endoscopic and radiological evidence of liver cirrhosis. Severity of

Table 1: Patient and disease characteristics

\begin{tabular}{|c|c|}
\hline Age & $51.56 \pm 11.50$ years \\
\hline Gender (Male: Female) & $3: 2$ \\
\hline Etiology of Cirrhosis & $\begin{array}{l}\text { Alcoholic Liver Disease - } 72 \%(n=36) \\
\text { Chronic Hepatitis B - } 10 \%(n=5) \\
\text { Non-Alcoholic Fatty Liver Disease - } 6 \%(n=3) \\
\text { Chronic Budd Chiari Syndrome }-6 \%(n=3) \\
\text { Hereditary Haemochromatosis }-2 \%(n=1) \\
\text { Cryptogenic Cirrhosis }-2 \%(n=1) \\
\text { Secondary Biliary Cirrhosis }-2 \%(n=1)\end{array}$ \\
\hline Indication for Hospital Admission & $\begin{array}{l}\text { Tense Ascites - } 40 \%(n=20) \\
\text { Variceal Bleeding - } 22 \%(n=11) \\
\text { Hepatic Encephalopathy - } 16 \%(n=8) \\
\text { Sponataneous Bacterial Peritonitis }-8 \%(n=4) \\
\text { Acute on Chronic Liver Failure }-6 \%(n=3) \\
\text { Acute Kidney Injury }-4 \%(n=2) \\
\text { Anemia - } 4 \%(n=2)\end{array}$ \\
\hline Duration of Liver Disease & $\begin{array}{l}<1 Y r-44 \%(n=22) \\
1-2 y r s-18 \%(n=9) \\
2-3 y r s-16 \%(n=8) \\
3-4 y r s-14 \%(n=7) \\
>4 y r s-8 \%(n=4)\end{array}$ \\
\hline Child Pugh's Score & $\begin{array}{l}A-0 \%(n=0) \\
B-42 \%(n=21) \\
C-58 \%(n=29)\end{array}$ \\
\hline MELD Na Score & $19.64 \pm 6$ \\
\hline Risk of Malnutrition (RFH-NPT) & $\begin{array}{l}\text { Low Risk }-18 \%(n=9) \\
\text { Intermediate risk }-28 \%(n=14) \\
\text { High Risk }-54 \%(n=27)\end{array}$ \\
\hline Rate of Malnutrition (MAMC $<5^{\text {th }}$ Percentile & $74 \%$ \\
\hline Rate of Fat Depletion (TST $<5^{\text {th }}$ Percentile) & $52 \%$ \\
\hline
\end{tabular}


Table 2. Nutritional parameters according to CTP score

\begin{tabular}{lccc}
\hline & Child B & Child C & p-value \\
\hline BMI & $23.5 \pm 4.08$ & 19.852 .59 & 0.001 \\
MAC & $24.98 \pm 4.14$ & $21.13 \pm 3.16$ & 0.001 \\
TSF & $1.09 \pm 0.47$ & 0.660 .30 & 0.001 \\
MAMC & $21.56 \pm 3.51$ & $19.01 \pm 2.73$ & 0.006 \\
\hline
\end{tabular}

the disease was determined by the Child Pugh's and Model for End Stage Liver Disease-Sodium (MELD $\mathrm{Na}$ ) scores. Sequential sampling was done and all consecutive patients meeting the inclusion criteria were included between April to June 2019. Among the 64 patients with liver cirrhosis who were admitted to the hospital during that time frame, 50 patients met the criteria for inclusion and were selected for the study.

The exclusion criteria were (i) Cirrhosis with coexisting tuberculosis, HIV or septicemia (ii) Comorbid conditions like heart failure and chronic kidney disease (iii) Coexisting hepatocellular carcinoma and other malignancies.

Nutritional assessment was done using the RFH-NPT and standard anthropometry. All assessments were done by the principle investigator to avoid any interobserver variations. RFH-NPT nutritional assessment tool is a simple tool that can be performed at the bedside. It is a semi-structured questionnaire based on the patients BMI, dietary intake and weight loss in the preceding 3 to 6 months. Based on these, patients are classified as having risks of malnutrition as low (0 points), moderate (1 point), or high (2-7 points). ${ }^{1,12,15}$

BMI was calculated in all patients. Since weight prior to fluid retention was not available in almost all patients with ascites, dry weight was estimated by subtracting the percentage of weight based upon severity of ascites (mild, 5\%; moderate, 10\%; severe, $15 \%)$, with an additional 5\% subtracted if bilateral pedal edema is present. 7,8 The MUAC was measured to the nearest centimeter using a measuring tape. The measurement was done on the right arm, midway between the tip of acromion and olecranon process. A skinfold caliper was used to measure the TST at the right arm to the nearest millimeter. An average of three measurements were taken for the MUAC and TST. MAMC was calculated by the formula: (MAMC $=$ MAC $-(3.145 x S F T) .{ }^{16}$ Malnutrition according to TST and MAMC were classified according to percentiles based on standard tables for age and sex and was defined by MAMC and/or TST $<5^{\text {th }}$ percentile. ${ }^{17}$

The study was approved by the Institutional Review Committee of Institute of Medicine, TUTH. Written informed consent was obtained from all patients prior to enrollment.

Statistical analysis was done using IBM SPSS Statistics version 23.0 software. Continuous variables
Table 3. Anthropometric measurements according to Nutritional Screening tools (RFH - NPT)

\begin{tabular}{lcccc}
\hline & $\begin{array}{c}\text { Low } \\
\text { Risk }\end{array}$ & $\begin{array}{c}\text { Moderate } \\
\text { Risk }\end{array}$ & $\begin{array}{c}\text { Severe } \\
\text { Risk }\end{array}$ & p-value \\
\hline BMI & $26.43 \pm 4.32$ & $21.07 \pm 2.88$ & $19.55 \pm 2.00$ & 0.000 \\
MAC & $27.88 \pm 3.81$ & $23.69 \pm 3.41$ & $20.55 \pm 2.44$ & 0.000 \\
TSF & $1.17 \pm 0.53$ & $0.97 \pm 0.43$ & $0.66 \pm 0.30$ & 0.002 \\
MAMC & $24.11 \pm 3.08$ & $20.58 \pm 2.96$ & $18.48 \pm 2.20$ & 0.000 \\
\hline
\end{tabular}

were expressed as means with standard deviation and analyzed using the independent student's t-test and one-way Anova. Categorical variables were analyzed using the chi-square test and Fischer's exact test where appropriate. A p-value of $<0.05$ was assumed to be statistically significant.

\section{RESULTS}

The mean age at presentation was $51.56 \pm 11.50$ years with a male to female ratio of $3: 2$. Alcohol related liver disease was the most common cause of cirrhosis found in $72 \%(n=36)$ of patients. $40 \%(n=20)$ of the patients were admitted for the management of tense ascites, the most frequent cause for hospital admission. Most patients, 44\% $(n=22)$ had a disease duration of less than 1 year. The severity of liver disease was classified according to the Child Pugh's score; $42 \%(n=21)$ and $58 \%(n=29)$ had Child B and C liver cirrhosis respectively. The average MELD Na score was $19.64 \pm 6$. Most patients, $54 \%(n=27)$ had high risk of malnutrition while $28 \%(n=14)$ and $18 \%$ ( $n=9$ ) had intermediate and low risks of malnutrition respectively in accordance with the RFH-NPT.

There was a significant difference in the average anthropometric measurements between Child B and $C$ cirrhotic patients (Table 2). Similar differences were also observed in the average anthropometric measurements between the risks of malnutrition using the RFH-NPT with statistical significance (Table 3).

Among the patients with Child C cirrhosis, 24 had severe risk of malnutrition while only five patients had intermediate risk. Among patients with Child $B$ cirrhosis, three patients had severe risk while nine patients each had moderate and mild risk. The difference in the risk of malnutrition between the two classes of cirrhosis was statistically significant with a $p$-value of $<0.05$. The prevalence of malnutrition

Table 4. RFH-NPT nutritional screening tool and Child Pugh's Score

\begin{tabular}{lccc}
\hline RFH-NPT & CTP B & CTP C & Total \\
\hline Mild & 9 & 0 & 9 \\
Moderate & 9 & 5 & 14 \\
Severe & 3 & 24 & 27 \\
Total & 21 & 29 & 50 \\
\hline
\end{tabular}


using the $\mathrm{MAMC}<5^{\text {th }}$ percentile was $74 \%$ of the total patients while depletion of fat stores i.e. TST $<5^{\text {th }}$ percentile was found in $52 \%$ of the patients.

\section{DISCUSSION}

According to our study, $82 \%(n=41)$ of the patients had some risk of malnutrition, moderate or severe using the RFH-NPT. The data is comparable to a similar study done in Assam, India where among the 123 patients included 90\% had malnutrition using the same tool. ${ }^{18}$ Other assessment tools like the Subjective Global Assessment (SGA) and The Royal Free Hospital-General Assessment (RFH-GA) can also correctly identify patients with malnutrition. However, these are time consuming and a skilled dietician is required. The RFH-NPT has been specifically designed for patients with liver disease and can be performed under three minutes even by non-specialist staff. In addition, it has a good correlation with the clinical deterioration, quality of life, a number of cirrhosisspecific complications including ascites, Hepatorenal Syndrome (HRS) and Hepatic Encephalopathy (HE) and transplant free survival of the patient. ${ }^{12}$ The risk of malnutrition using this tool is significantly higher in patients with Child $\mathrm{C}$ cirrhosis as compared to Child B in our study showing that it corroborates well with the severity of liver cirrhosis. BMI provides a simple and accurate method of assessing nutrition. However, it is usually overestimated in patients with ascites. In a study including 396 hospitalized cirrhotic patients, BMI was significantly overestimated in patients with tense ascites. ${ }^{19}$ However, we calculated the patients BMI after modifying the weights according to the amount of ascites or edema. Our study shows that the average modified BMI was significantly different among patients with Child B and C cirrhosis as well as those with Mild, Moderate or severe risk of malnutrition.

In a study of 212 patients from Italy, 37\% of patients had protein calorie malnutrition (MAMC $<5^{\text {th }}$ percentile) while $28 \%$ had body fat depletion (TST $<5^{\text {th }}$ percentile). ${ }^{20}$ A similar study from France including 396 patients also found $53.2 \%$ and $38.2 \%$ of patients with MMAC and TST below the $5^{\text {th }}$ percentile. ${ }^{21}$ A Malaysian study has shown similar findings with $\mathrm{MAMC}<5^{\text {th }}$ percentile among 50\% and TST $<5^{\text {th }}$ percentile among $30 \% .{ }^{22}$ However, the rates of malnutrition and fat depletion was much higher in our study with a MAMC $<5^{\text {th }}$ percentile among $74 \%$ of patients and TST $<5^{\text {th }}$ percentile among $52 \%$ of the patients. These data show that malnutrition in cirrhosis is a global issue. The higher rates among Nepalese patients is probably due to poorer socioeconomic status.

The anthropometric measurements in our study including MUAC, MAMC and TST corroborated well with the Child Pugh's Class as well as risk of malnutrition determined by the RFH-NPT. These findings are consistent with similar studies done in India and Malaysia showing that anthropometric measurements correlate well with the severity of liver cirrhosis as well as malnutrition. ${ }^{18,22}$ An Italian study evaluating nutrition and survival among cirrhotic patients has shown that patients with a MAMC and TST below the $5^{\text {th }}$ percentile have a significantly lower survival at 6, 12 and 24 months. ${ }^{20}$ The authors of that study including 212 patients have also suggested that MAMC and TST could be incorporated in the Child Pugh's Score to increase the predictive value of this score. In a study among 102 liver transplant recipients, patients with a lower TST and MAMC had higher rates of post-operative bacterial infections and abnormal liver function. ${ }^{23}$

In patients with liver cirrhosis, sarcopenia or loss of skeletal muscle mass is the major component of malnutrition. Multiple mechanisms including decreased dietary intake, increased skeletal muscle ammonia, decrease in growth hormone, testosterone and branched chain aminoacids as well endotoxemia have all been implicated in the pathogenesis of sarcopenia. 8,15,24,25 The best and most accurate methods to assess sarcopenia are radiology with $\mathrm{CT}$, MRI or DEXA. However, these tests are expensive, not readily available and in case of $\mathrm{CT}$, there are high risks of exposure to contrast and ionizing radiation. Studies have shown that anthropometric measurements like MAC and MAMC correspond well to sarcopenia. ${ }^{15,26}$ Hence, anthropometry can be used as a more practical means to evaluate sarcopenia especially when repeated assessments may be required.

This study has some limitations. Since the sample size was small, the findings of this study may not be generalized to the wider prevalence of liver cirrhosis. While measuring the BMl the true weight of most patients could not be used due presence of fluid overload. We did use estimated weights using standard recommendations but this could have influenced our BMI measurements. We included only hospitalized patients with decompensated liver cirrhosis who are known to be at an increased risk of malnutrition. Hence, the true prevalence of malnutrition in our context may have been slightly overestimated. Nevertheless, this is the first kind of study evaluating the nutritional status among Nepalese patients with liver cirrhosis. The high rate of malnutrition among these patients indicate that nutritional assessment and nutritional rehabilitation should be an integral part of standard medical treatment in liver cirrhosis. It also serves as a basis to conduct larger studies including a more heterogenous population set of patients with liver cirrhosis.

\section{CONCLUSION}

Malnutrition is a common complication among patients with liver cirrhosis. Nutritional assessment 
using RFH-NPT and anthropometry correlate well with the severity of malnutrition and the patients Child Pugh Score. Hence, they can be used as a valuable tools for screening for malnutrition as well as prognostication of patients with liver cirrhosis.

\section{CONFLICT OF INTEREST}

None declared.

\section{REFERENCES}

1. Amodio P, Bemeur C, Butterworth R, Cordoba J, Kato $A$, Montagnese $S$, et al. The nutritional management of hepatic encephalopathy in patients with cirrhosis: International society for hepatic encephalopathy and nitrogen metabolism consensus. Hepatology. 2013 Jul;58(1):325-36.

2. Cheung $K$, Lee SS, Raman M. Prevalence and Mechanisms of Malnutrition in Patients With Advanced. YJCGH. 2012;10(2):117-25.

3. Mendenhall C, Roselle GA, Gartside P, Moritz T. Relationship of Protein Calorie Malnutrition to Alcoholic Liver Disease : A Reexamination of Data from Two Veterans Administration Cooperative Studies. $1995 ; 19(3)$

4. Sam J, Nguyen GC. Protein-calorie malnutrition as a prognostic indicator of mortality among patients hospitalized with cirrhosis and portal hypertension. Liver Int. 2009 Oct;29(9):1396-402.

5. Huisman EJ, Trip EJ, Siersema PD, van Hoek B, van Erpecum KJ. Protein energy malnutrition predicts complications in liver cirrhosis. Eur J Gastroenterol Hepatol. 2011 Nov;23(11):982-9.

6. Merli M, Lucidi C, Giannelli V, Giusto M, Riggio O, Falcone $M$, et al. Cirrhotic Patients Are at Risk for Health Care-Associated Bacterial Infections. Clin Gastroenterol Hepatol. 2010 Nov;8(11):979-985.

7. Tandon P, Raman M, Mourtzakis M, Merli M. A practical approach to nutritional screening and assessment in cirrhosis. Hepatology. 2017;65(3):1044-57.

8. Merli M, Berzigotti A, Zelber-Sagi S, Dasarathy S, Montagnese S, Genton L, et al. EASL Clinical Practice Guidelines on nutrition in chronic liver disease. J Hepatol. 2018

9. Charney P. Nutrition Screening vs Nutrition Assessment: How Do They Differ? Nutr Clin Pract 2008 Aug;23(4):36672

10. Lochs $H$, Allison $S P$, Meier $R$, Pirlich M, Kondrup $J$, Schneider S, et al. Introductory to the ESPEN Guidelines on Enteral Nutrition: Terminology, Definitions and General Topics. Clin Nutr [Internet]. 2006;25(2):180-6.

11. The Development And Validation of A Nutritional Prioritising Tool.... EASL LiverTreeTM. Arora S. Apr 20 2012; 18793.
12. Borhofen SM, Gerner $C$, Lehmann J, Fimmers R, Görtzen J, Hey B, et al. The Royal Free Hospital-Nutritional Prioritizing Tool Is an Independent Predictor of Deterioration of Liver Function and Survival in Cirrhosis. Dig Dis Sci. 2016 Jun 2;61(6):1735-43.

13. Morgan MY, Madden AM, Soulsby CT. Derivation and validation of a new global method for assessing nutritional status in patients with cirrhosis. Hepatology.2006 Oct;44(4):823-35.

14. Alberino F, Gatta A, Amodio P, Merkel C, Di Pascoli L, Boffo $G$, et al. Nutrition and survival in patients with liver cirrhosis. Nutrition. 2001 Jun 1;17(6):445-50.

15. Tandon $P$, Low G, Mourtzakis $M$, Zenith L, Myers RP, Abraldes JG, et al. A Model to Identify Sarcopenia in Patients With Cirrhosis. Clin Gastroenterol Hepatol. 2016 Oct;14(10):1473-1480.

16. Jones JM. Reliability of nutritional screening and assessment tools. Nutrition. 2004 Mar ; 20(3):307-11.

17. Frisancho AR. New norms of upper limb fat and muscle areas for assessment of nutritional status. Am J Clin Nutr. 1981 Nov 1;34(11):2540-5.

18. Begum T.A Comparative Assesment Of Nutritional Status In Cirrhosis Of Liver By Anthropometry And RFNP-Tool In Indian Population.Indian Journal of applied science. 2017;7(6):236-239.

19. Campillo B, Paillaud E, Uzan I, Merlier I, Abdellaoui M. Value of body mass index in the detection of severe malnutrition : influence of the pathology and changes in anthropometric parameters. 2004;551-9.

20. Alberino F, Gatta A, Amodio P, Merkel C, Di Pascoli L, Boffo $G$, et al. Nutrition and survival in patients with liver cirrhosis. Nutrition. 2001 Jun;17(6):445-50.

21. Campillo B, Richardet J-P, Scherman E, Bories PN. Evaluation of nutritional practice in hospitalized cirrhotic patients: results of a prospective study. Nutrition . 2003 Jun;19(6):515-21.

22. Tai MLS, Goh KL, Mohd-Taib SH,et al. Anthropometric, biochemical and clinical assessment of malnutrition in Malaysian patients with advanced cirrhosis. Nutr J. 2010;9(1):1-7.

23. Harrison J, McKiernan J, Neuberger JM. A prospective study on the effect of recipient nutritional status on outcome in liver transplantation. Transpl Int. 1997;10(5):369-74.

24. Dasarathy S, Merli M. Sarcopenia from mechanism to diagnosis and treatment in liver disease. J Hepatol. 2016;65(6):1232-44.

25. Tessari P. Protein metabolism in liver cirrhosis: from albumin to muscle myofibrils. Curr Opin Clin Nutr Metab Care. 2003 Jan 1;6(1):79-85

26. Santos $L A A$, Lima $T B$, letsugu $M$ do $V$, Nunes $H R$ de $C$, Qi X, Romeiro FG. Anthropometric measures associated with sarcopenia in outpatients with liver cirrhosis. Nutr Diet. 2019 Mar 14. doi.wiley.com/10.1111/17470080.12523 